\title{
Effect of perioperative $\beta$ blockade in patients with diabetes undergoing major non-cardiac surgery: randomised placebo controlled, blinded multicentre trial
}

\author{
Anne Benedicte Juul, Jørn Wetterslev, Christian Gluud, Allan Kofoed-Enevoldsen, Gorm Jensen, Torben Callesen, \\ Peter Nørgaard, Kim Fruergaard, Morten Bestle, Rune Vedelsdal, André Miran, Jon Jacobsen, Jakob Roed, Maj-Britt \\ Mortensen, Lise Jørgensen, Jørgen Jørgensen, Marie-Louise Rovsing, Pernille Lykke Petersen, Frank Pott, Merete \\ Haas, Rikke Albret, Lise Lotte Nielsen, Gun Johansson, Pia Stjernholm, Yvonne Mølgaard, Nikolai Bang Foss, Jeanie \\ Elkjær, Bjørn Dehlie, Klavs Boysen, Dusanka Zaric, Anne Munksgaard, Jørn Bo Madsen, Bjarne Øberg, Boris \\ Khanykin, Tine Blemmer, Stig Yndgaard, Grazyna Perko, Lars Peter Wang, Per Winkel, Jørgen Hilden, Per Jensen, \\ Nader Salas, and the DIPOM Trial Group
}

\begin{abstract}
Objectives To evaluate the long term effects of perioperative $\beta$ blockade on mortality and cardiac morbidity in patients with diabetes undergoing major non-cardiac surgery.

Design Randomised placebo controlled and blinded multicentre trial. Analyses were by intention to treat. Setting University anaesthesia and surgical centres and one coordinating centre.

Participants 921 patients aged $>39$ scheduled for major non-cardiac surgery.

Interventions $100 \mathrm{mg}$ metoprolol controlled and extended release or placebo administered from the day before surgery to a maximum of eight perioperative days.

Main outcome measures The composite primary outcome measure was time to all cause mortality, acute myocardial infarction, unstable angina, or congestive heart failure.

Secondary outcome measures were time to all cause mortality, cardiac mortality, and non-fatal cardiac morbidity.

Results Mean duration of intervention was 4.6 days in the metoprolol group and 4.9 days in the placebo group.

Metoprolol significantly reduced the mean heart rate by $11 \%$ (95\% confidence interval 9\% to 13\%) and mean blood pressure by $3 \%(1 \%$ to $5 \%)$. The primary outcome occurred in 99 of 462 patients in the metoprolol group (21\%) and 93 of 459 patients in the placebo group (20\%) (hazard ratio $1.06,0.80$ to 1.41 ) during a median follow-up of 18 months (range 6-30). All cause mortality was $16 \%(74 / 462)$ in the metoprolol group and $16 \%$ $(72 / 459)$ in the placebo group (1.03, 0.74 to 1.42$)$. The difference in risk for the proportion of patients with serious adverse events was $2.4 \%$ ( $-0.8 \%$ to $5.6 \%)$.

Conclusions Perioperative metoprolol did not significantly affect mortality and cardiac morbidity in these patients with diabetes. Confidence intervals, however, were wide, and the issue needs reassessment.

Trial registration Current Controlled Trials ISRCTN58485613.
\end{abstract}

\section{Introduction}

Cardiac morbidity and mortality after non-cardiac surgery is $11 \%$ to $34 \%$ in patients with diabetes, recent myocardial infarction, unstable angina, or congestive heart failure. ${ }^{1-4}$ Complications are probably due to perioperative myocardial ischaemia. $^{15}$

Perioperative $\beta$ blockade is recommended in patients at cardiac risk undergoing major non-cardiac surgery. ${ }^{6}$ The recommendations are based on the results of small trials indicating that patients at cardiac risk should receive perioperative $\beta$ blockade when they undergo major non-cardiac surgery. ${ }^{2578}$ Devereaux et al carried out a meta-analysis of trials on perioperative $\beta$ blockers up to April 2003. ${ }^{8}$ They found insufficient evidence for a reduction of major cardiovascular events. ${ }^{8}$ Subsequently, the perioperative $\beta$ blockade (POBBLE) trial failed to show significant effects of perioperative $\beta$ blockade on mortality and major cardiovascular events in patients undergoing vascular surgery. ${ }^{9}$

The multicentre study group of perioperative ischaemia suggested that diabetes was a significant predictor of postoperative death. ${ }^{5}$ Perioperative $\beta$ blockade in patients with diabetes and additional risk factors for coronary artery disease seemed beneficial, with a two year hazard ratio for death of 0.25 $(\mathrm{P}=0.03) .^{7}$ The American College of Cardiology and the American Heart Association assert that patients with diabetes have the same risk as coronary artery disease patients ${ }^{6}$ and therefore may benefit from perioperative $\beta$ blockade. ${ }^{10}{ }^{11}$ We conducted the diabetes postoperative mortality and morbidity (DIPOM) trial to assess metoprolol versus placebo for patients with diabetes undergoing major non-cardiac surgery. ${ }^{10}$

\section{Methods}

The DIPOM trial is an investigator initiated and controlled, randomised placebo controlled, multicentre trial with central randomisation and blinding of all parties in all phases. ${ }^{10}$ The code was broken when analyses were completed and a conclusion formulated. 


\section{Organisation}

Thirteen anaesthesia and surgical centres in nine hospitals in the greater Copenhagen area participated. An independent and blinded event committee adjudicated all reports of outcomes registered in public databases. An independent and blinded data monitoring and safety committee assessed the interim analyses. ${ }^{10}$ The Copenhagen trial unit coordinated randomisation, collection, and distribution of patients' records to the event and safety committees, data entry, and data management. Clean files were exported to the department of biostatistics, University of Copenhagen, for analyses.

\section{Patient recruitment}

Between 1 July 2000 and 1 July 2002 project nurses contacted eligible patients aged $>39$ with diabetes who were scheduled for major (that is, expected duration over one hour) non-cardiac surgery. Patients with either insulin or non-insulin dependent diabetes were included. ${ }^{12}$ All participants gave written informed consent. Exclusion criteria were: no written informed consent; systemic treatment with $\beta$ blocker; conditions indicating $\beta$ blocker treatment; New York Heart Association class IV congestive heart failure; third degree atrioventricular blockade; pregnancy or breast feeding; allergic to metoprolol or placebo; or previously included in the DIPOM trial. ${ }^{10}$

\section{Randomisation}

A computer generated the allocation sequence and served a telephone voice response randomisation system. The allocation sequence was in blocks of eight and stratified for centre, age $>65$, sex, expected perioperative stress (high and intermediate risk or low risk surgery), history of coronary artery disease, and active malignant disease. ${ }^{10}$ Patients were randomly assigned to metoprolol succinate controlled/extended release (CR/XL) or matching placebo. When possible patients were given a test dose of $50 \mathrm{mg}$ study drug the evening before surgery (day 1). If it was tolerated, they were given two $50 \mathrm{mg}$ tablets (full daily dose) at least two hours before induction of anaesthesia on the day of surgery (day 2). The study drug was administered once daily until discharge from the hospital or to a maximum of eight days. Half dose was given in patients with a heart rate of 55-65 beats per minute and systolic blood pressure $\geq 100 \mathrm{~mm} \mathrm{Hg}$. The study drug was withheld in patients with a heart rate $<55$ beats per minute or systolic blood pressure $<100 \mathrm{~mm} \mathrm{Hg}$. When oral administration was not feasible, $5 \mathrm{mg}$ metoprolol or matching placebo was given intravenously before surgery and every sixth hour. Incorrect dosing of trial drug was considered a protocol violation.

\section{Follow-up}

We collected morbidity and mortality data from the Danish national hospital register, which contains information about all somatic hospital admissions in Denmark, ${ }^{10}$ and the centralised civil register, which records the vital status of all inhabitants in Denmark..$^{10}$ In addition, all patients were recalled six months after discharge, when we recorded use of $\beta$ blockers after discharge and an electrocardiogram.

\section{Outcome measures}

The primary outcome measure was time to the composite outcome of all cause mortality, acute myocardial infarction, unstable angina, or congestive heart failure discovered or aggravated during admission to hospital. Non-fatal myocardial infarction was diagnosed using electrocardiography; concentrations of troponin, CK-MB, or LDH-1 isoenzymes; and pain..$^{10}$ The criteria to be fulfilled were a rise in concentrations of troponin, $\mathrm{LDH}-1$, or CK-MB at or above double the upper normal concentration and presence of angina, equivalent of angina, respiratory distress, palpitations, atypical pain, sickness, or development of $Q$ wave, ST elevation, ST depression, or T wave abnormalities. At the outpatient visit we recorded the presence of new $Q$ waves according to the Minnesota Code Criteria ${ }^{10}$ as myocardial infarction. Cardiac heart failure was defined as start of medication, increased dose of existing medication, or new class of medication for cardiac heart failure. Unstable angina pectoris was diagnosed

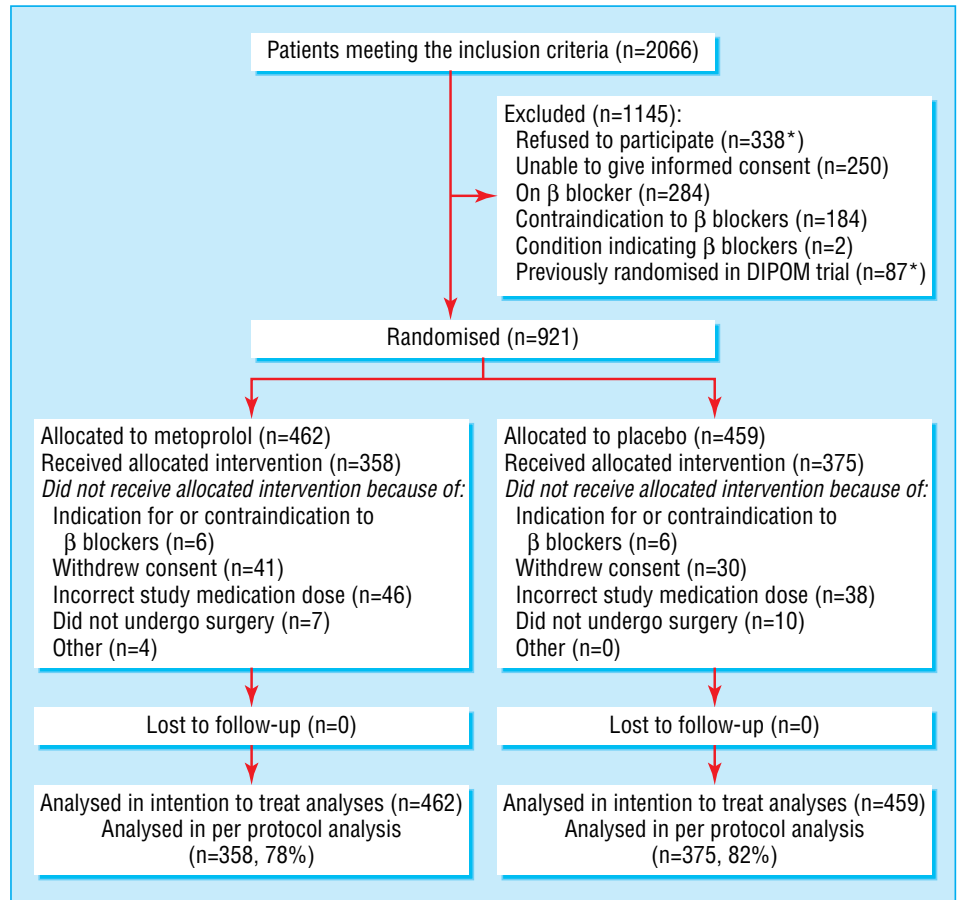

Fig 1 Flow of patients through study ( ${ }^{*}$ includes five patients who refused to take part after randomisation but before any medication and surgery; fincludes two patients who had previously been randomised in the DIPOM trial) 
if the patient had a record of typical pain and no detectable rise in coronary enzymes along with a specific medical intervention for unstable angina pectoris or ST depression or inversion of T wave. Secondary outcomes were all cause mortality, cardiac mortality, non-cardiac mortality, and cardiac morbidity. ${ }^{10}$ We registered serious adverse events and adverse events leading to withdrawal of the study drug.

\section{Statistical analysis}

We estimated that $30 \%$ of patients in the placebo group would have a primary outcome due to a shorter follow-up, inclusion of younger patients, and the wide $95 \%$ confidence interval in previous studies. ${ }^{711}$ To detect a 33\% relative risk reduction in the metoprolol group we needed a sample size of 824 patients with a two sided $\alpha=0.05$ and power $(1-\beta)=0.90$. We aimed to randomise 1000 patients. ${ }^{10}$

We compared Kaplan-Meier survival curves by log rank test. To estimate the intervention effect, we used univariate Cox regression models as the primary analyses. Adjusted intervention effects were calculated with all variables used for stratification during randomisation in a multivariate Cox regression analysis. ${ }^{13}$ We also calculated cumulated intensities, time dependent effects, and residual plots substantiating proportional hazards. We performed a prespecified subgroup analysis of the patients fulfilling the inclusion criteria in the trial of Mangano et al (having at least one risk factor for coronary artery disease besides diabetes and undergoing high risk or intermediate risk surgery $\left.^{711}\right)$. $\mathrm{P}<0.05$ was considered significant.

\section{Results}

We randomised 921 patients: 462 to metoprolol and 459 to placebo. All patients were followed up until 1 January 2003 and analysed in intention to treat analyses; 733 patients (80\%) were analysed in per protocol analyses (fig 1). Baseline and surgical characteristics were comparable in the two groups (table 1). In addition to diabetes, 496 of the patients (54\%) had a history of, or an additional risk factor for, coronary artery disease and were undergoing high risk or intermediate risk surgery. The median follow-up was 18 months (range 6-30 months). At six months, 720 patients $(78 \%)$ had an outpatient follow-up, and 713 patients $(77 \%)$ underwent electrocardiography. Seven patients in the metoprolol group and five in the placebo group had received $\beta$ blockers during follow-up.

\section{Primary outcome}

Overall 99 of 462 patients in the metoprolol group (21\%) and 93 of 459 patients in the placebo group $(20 \%)$ had a primary outcome. Figure 2 shows the Kaplan-Meier estimates of the primary outcome measure in the two groups (log rank test $\mathrm{P}=0.66)$. Within 30 days postoperatively 27 of 462 patients in the metoprolol group $(6 \%, 95 \%$ confidence interval $4 \%$ to $8 \%$ ) and 21 of 459 patients in the placebo group (5\%, 3\% to $7 \%$ ) had a primary outcome.

Table 2 shows predictors of the primary outcome in univariate Cox regression models. We found no significant effect of metoprolol compared with placebo (hazard ratio $1.06,0.80$ to $1.41, \mathrm{P}=0.66$ ) and no evidence of significant variation in treatment effect according to centre or baseline characteristics. Presence of coronary artery disease or malignant disease contained significant prognostic information. Multivariate analysis with all variables used for stratification in the model showed no significant effect of metoprolol (1.05, 0.79 to 1.40) (table 2). Metoprolol still had no significant effect even when we added preoperative insulin treatment to the multivariate model (1.07,
0.80 to 1.42 ) (table 2). The effect of preoperative insulin treatment was not significant in a univariate analysis $(\mathrm{P}=0.46)$. In the subgroup of 496 patients fulfilling the criteria of Mangano et

Table 1 Entry characteristics of patients randomised to metoprolol or placebo. Figures are number (percentage) of patients unless stated otherwise

\begin{tabular}{|c|c|c|}
\hline & Metoprolol $(n=462)$ & Placebo $(n=459)$ \\
\hline \multicolumn{3}{|l|}{ Demographics } \\
\hline Mean (SD) age (years) & $64.9(11.1)$ & $64.8(110.8)$ \\
\hline Mean (SD) body mass index & $27.2(5.4)$ & $26.9(5.2)$ \\
\hline No $(\%)$ of women & $191(41)$ & $191(42)$ \\
\hline \multicolumn{3}{|l|}{ Cardiovascular disease } \\
\hline Congestive heart failure & $47(10)$ & 48 (11) \\
\hline Atrial fibrillation & $34(7)$ & $38(8)$ \\
\hline Arrhythmia requiring treatment & $12(3)$ & $13(3)$ \\
\hline Angina pectoris & $56(12)$ & 48 (11) \\
\hline Previous acute myocardial infarction & $36(8)$ & $34(7)$ \\
\hline Previous PTCA/CABG & $20(4)$ & $15(3)$ \\
\hline History of hypertension & $254(55)$ & $288(58)$ \\
\hline Calcium channel blockers & $70(15)$ & $81(18)$ \\
\hline Diuretics & $155(34)$ & $170(37)$ \\
\hline ACE inhibitor & $130(28)$ & $148(32)$ \\
\hline Anticoagulants & $114(25)$ & $99(22)$ \\
\hline Statins & $53(12)$ & $46(10)$ \\
\hline Angiotensin II receptor blocker & $29(6)$ & $27(6)$ \\
\hline $\begin{array}{l}\text { History of coronary artery disease and } \\
\text { hypertension }\end{array}$ & $279(61)$ & $286(62)$ \\
\hline \multicolumn{3}{|l|}{ Antidiabetic treatment } \\
\hline Diet alone & $81(18)$ & $80(17)$ \\
\hline Oral hypoglycaemic agent & $201(44)$ & 191 (42) \\
\hline Insulin & $153(33)$ & $164(36)$ \\
\hline Insulin and oral hypoglycaemic agent & $26(6)$ & $24(5)$ \\
\hline \multicolumn{3}{|l|}{ Diabetes duration and complications } \\
\hline Mean (SD) duration of diabetes (years) & $12.0(11.8)$ & $11.6(11)$ \\
\hline Diabetic neuropathy & $116(25)$ & $124(27)$ \\
\hline Diabetic retinopathy & $92(20)$ & $85(19)$ \\
\hline Diabetic nephropathy & $35(8)$ & $37(8)$ \\
\hline \multicolumn{3}{|l|}{ Concurrent diseases and surgical risk } \\
\hline Current smoker & $179(39)$ & $171(38)$ \\
\hline Former smoker & $183(40)$ & $169(37)$ \\
\hline Excessive alcohol consumption & $25(5)$ & $28(6)$ \\
\hline Active malignant disease & $86(19)$ & $88(19)$ \\
\hline \multicolumn{3}{|l|}{ Surgical risk } \\
\hline Expected high or intermediate risk & $283(61)$ & $278(61)$ \\
\hline Expected low risk surgery & $179(39)$ & $181(39)$ \\
\hline \multicolumn{3}{|l|}{ Type of surgery } \\
\hline Orthopaedic & $154(33)$ & 149 (32) \\
\hline Intra-abdominal & $126(27)$ & $129(28)$ \\
\hline Neurological & $40(9)$ & $32(7)$ \\
\hline Vascular & $30(7)$ & $32(7)$ \\
\hline Gynaecological & $23(5)$ & $24(5)$ \\
\hline Thoracic & $20(4)$ & $17(4)$ \\
\hline Other* & $69(15)$ & $76(17)$ \\
\hline \multicolumn{3}{|l|}{ Type of anaesthesia } \\
\hline General & $269(58)$ & $295(64)$ \\
\hline Combined epidural and general & $85(18)$ & $78(17)$ \\
\hline Epidural or spinal & $91(20)$ & $69(15)$ \\
\hline Unknown & $17(4)$ & $17(4)$ \\
\hline \multicolumn{3}{|l|}{ Duration of surgery } \\
\hline Mean hours (range) $\dagger$ & $2.6(0.5-9.8)$ & $2.6(0.4-9.8)$ \\
\hline \multicolumn{3}{|l|}{ Blood transfusion } \\
\hline No transfused & $33(8)$ & $38(9)$ \\
\hline Mean (SD) amount transfused (ml) & 809 (735) & $825(528)$ \\
\hline
\end{tabular}

ACE inhibitor=angiotensin converting enzyme inhibitor; PTCA/CABG=percutaneous transluminal coronary angioplasty/coronary artery bypass graft.

*Includes head and neck surgery, plastic surgery, eye surgery, or general surgery.

†Measured from induction of anaesthesia to time of final blood pressure measurement. 
$\mathrm{al}^{7}$ there was no significant effect of metoprolol $(1.03,0.71$ to 1.50). The per protocol analysis and analyses of risk of type of surgery yielded similar results.

\section{Mortality and cardiac morbidity}

There were no significant differences between the intervention groups in any of the secondary outcomes. Seventy four of 462 patients $(16 \%)$ in the metoprolol group and 72 of 459 in the placebo group (16\%) died (1.03, 0.74 to 1.42$)$. In a multivariate analysis with all stratification variables in the model the hazard ratio of death was 1.01 ( 0.72 to 1.41$)$. Addition of preoperative insulin treatment to the multivariate analysis changed the hazard ratio of the intervention effect to 1.03 (0.74 to 1.42), and the effect of preoperative insulin treatment was not significant $(\mathrm{P}=0.068)$. Forty six of 462 patients in the metoprolol group and 45 of 459 in the placebo group had a cardiac outcome (1.02, 0.67 to 1.67 ), with an adjusted hazard ratio of 1.03 (0.69 to 1.54 ). We found similar non-significant effects of metoprolol on cardiac mortality, non-fatal cardiac morbidity, or non-cardiac mortality (table 3).

\section{Safety outcomes}

The administration of metoprolol was associated with a significant increase in low heart rate and blood pressure; 147 of 462 patients in the metoprolol group (32\%) and 80 of 459 in the placebo group $(17 \%)$ had episodes of heart rate $<65$ beats per minute or systolic blood pressure $<100 \mathrm{~mm} \mathrm{Hg}$ (table 4). Serious adverse events occurred in $8 \%$ in the metoprolol group and $5 \%$ in the placebo group, a risk difference of $2.4 \%(-0.8 \%$ to $5.6 \%)($ table 5).

\section{Compliance and haemodynamics}

In total 358 patients in the metoprolol group and 375 in the placebo group received the as intended intervention; one or more
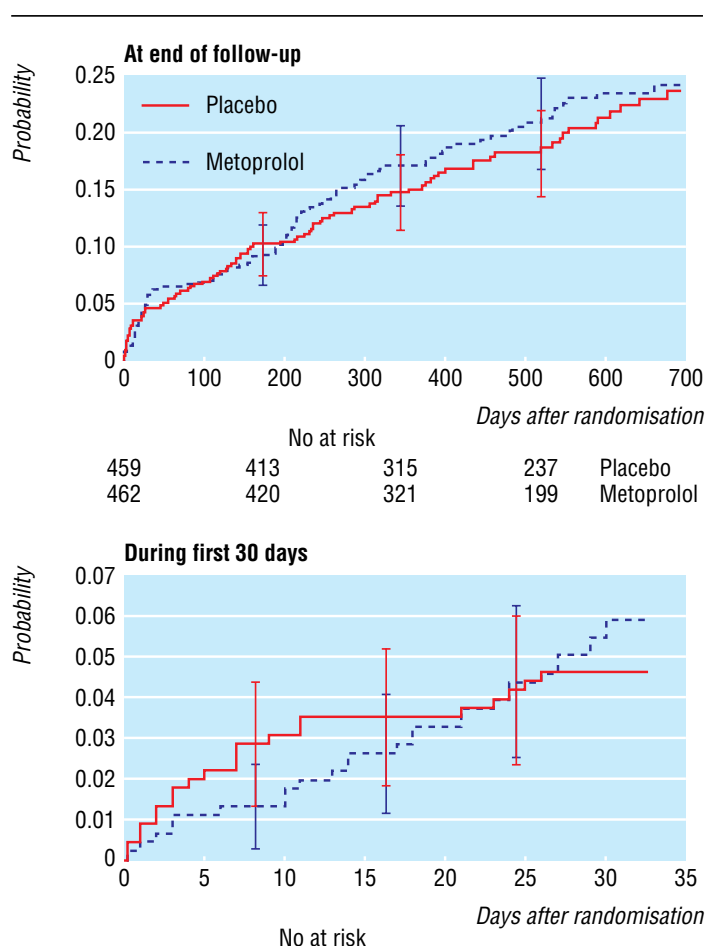

\begin{tabular}{lllll}
\multicolumn{5}{c}{ No at risk } \\
459 & 449 & 445 & 441 & Placebo \\
462 & 457 & 452 & 444 & Metoprolol
\end{tabular}

Fig 2 Kaplan-Meier plot of time to primary outcome measure during maximum follow-up and during first 30 days (with $95 \%$ confidence intervals)
Table 2 Intention to treat analysis. Predictors of primary outcome (all cause mortality, acute myocardial infarction, unstable angina, and congestive heart failure) among patients with diabetes undergoing non-cardiac surgery

\begin{tabular}{|c|c|c|}
\hline & Hazard ratio $(95 \% \mathrm{CI})$ & $P$ value \\
\hline \multicolumn{3}{|l|}{ Univariate analyses } \\
\hline Metoprolol & 1.06 (0.80 to 1.41$)$ & 0.66 \\
\hline Age (>65 or $\leq 65$ years) & 2.62 (1.91 to 3.58 ) & $<0.001$ \\
\hline Sex & $1.25(0.93$ to 1.68$)$ & 0.13 \\
\hline Coronary artery disease & 1.60 (1.18 to 2.17 ) & 0.002 \\
\hline Malignant disease & $1.90(1.40$ to 2.59$)$ & $<0.001$ \\
\hline Expected surgical stress & 1.31 (0.98 to 1.77$)$ & 0.07 \\
\hline Centre & Not shown† & 0.08 \\
\hline \multicolumn{3}{|l|}{ Multivariate analyses* } \\
\hline Metoprolol & $1.05(0.79$ to 1.40$)$ & 0.53 \\
\hline Age (>65 or $\leq 65$ years) & 2.48 (1.79 to 3.43 ) & $<0.001$ \\
\hline Sex & 1.17 (0.86 to 1.57$)$ & 0.31 \\
\hline Coronary artery disease & 1.35 (0.99 to 1.85$)$ & 0.056 \\
\hline Malignant disease & 1.55 (1.09 to 2.19 ) & 0.014 \\
\hline Expected surgical stress & $1.02(0.39$ to 1.41$)$ & 0.89 \\
\hline Centre & Not shown† & 0.14 \\
\hline \multicolumn{3}{|c|}{ Multivariate analyses including preoperative insulin treatment } \\
\hline Metoprolol & 1.07 (0.80 to 1.42$)$ & 0.65 \\
\hline Age (>65 or $\leq 65$ years) & 2.56 (1.85 to 3.55 ) & $<0.001$ \\
\hline Sex & 1.17 (0.87 to 1.59$)$ & 0.30 \\
\hline Coronary artery disease & 1.38 (1.01 to 1.88$)$ & 0.04 \\
\hline Malignant disease & 1.57 (1.10 to 2.22) & 0.01 \\
\hline Expected surgical stress & 1.04 (0.75 to 1.44$)$ & 0.83 \\
\hline Centre & Not shown† & 0.11 \\
\hline Preoperative insulin treatment & 1.36 (1.02 to 1.83$)$ & 0.04 \\
\hline
\end{tabular}

*Based on a Cox proportional hazards model including all variables used for stratification at randomisation and trial drug as mandatory covariates.

fData not reported from all 13 centres.

Table 3 Intention to treat analysis. Hazard ratios of effect of perioperative $\beta$ blockade on secondary outcomes among patients with diabetes undergoing non-cardiac surgery

\begin{tabular}{|c|c|c|}
\hline & Hazard ratio* $(95 \% \mathrm{Cl})$ & $P$ value \\
\hline \multicolumn{3}{|l|}{ Univariate analyses } \\
\hline All cause mortality & 1.03 (0.74 to 1.42$)$ & 0.88 \\
\hline Cardiac events & $1.02(0.67$ to 1.57$)$ & 0.91 \\
\hline Cardiac death & 0.85 (0.45 to 1.60$)$ & 0.61 \\
\hline Non-fatal cardiac events & $1.24(0.70$ to 2.17$)$ & 0.46 \\
\hline Non-cardiac death & 1.10 (0.75 to 1.61$)$ & 0.63 \\
\hline \multicolumn{3}{|l|}{ Multivariate analyses } \\
\hline All cause mortality & 1.01 (0.72 to 1.41$)$ & 0.79 \\
\hline Cardiac events & 1.03 (0.69 to 1.54$)$ & 0.87 \\
\hline Cardiac death & 0.84 (0.46 to 1.52$)$ & 0.56 \\
\hline Non-fatal cardiac events & 1.23 (0.68 to 2.23$)$ & 0.44 \\
\hline Non-cardiac death & $1.13(0.75$ to 1.70$)$ & 0.65 \\
\hline
\end{tabular}

${ }^{*}$ Adjusted in multivariate analysis for all variables used for stratification at randomisation and trial drug as mandatory covariates.

minor protocol violations occurred in 104 patients in the metoprolol group and 84 in the placebo group (fig 1).

The test dose was administered the day before surgery in 678 patients (74\%). The target dose thereafter was $100 \mathrm{mg}$ metoprolol or placebo daily. Several patients received either half the dose or no study drug because of low blood pressure or low heart rate (table 4). The mean duration of drug administration was 4.6 (range 0 to 8 ) days in the metoprolol group and 4.9 (0 to 8 ) days in the placebo group.

The heart rate and blood pressure were measured immediately before the next dose of study drug (table 6 ). Before the first dose of study drug, the mean heart rate and mean arterial pressure did not differ between the two groups. Thereafter, patients in the metoprolol group had significantly lower mean 
Table 4 Daily dose of metoprolol (M) and placebo (P). Figures are number (percentage) of patients

\begin{tabular}{|c|c|c|c|c|c|c|c|c|c|c|c|c|c|c|}
\hline \multirow{2}{*}{ Variable } & \multicolumn{2}{|c|}{ Day 2* } & \multicolumn{2}{|c|}{ Day 3} & \multicolumn{2}{|c|}{ Day 4} & \multicolumn{2}{|c|}{ Day 5} & \multicolumn{2}{|c|}{ Day 6} & \multicolumn{2}{|c|}{ Day 7} & \multicolumn{2}{|c|}{ Day 8} \\
\hline & M & $P$ & $M$ & $\mathbf{P}$ & M & $P$ & $M$ & $P$ & M & $\mathbf{P}$ & M & $P$ & M & $\mathbf{P}$ \\
\hline Full dose & 294 (64) & 357 (78) & 311 (67) & 358 (78) & $235(51)$ & $304(66)$ & $192(42)$ & 267 (58) & $167(36)$ & $231(50)$ & $145(31)$ & $202(44)$ & $123(27)$ & $173(38)$ \\
\hline Half dose† & $122(26)$ & 70 (15) & 65 (14) & $32(7)$ & 58 (13) & $31(7)$ & 57 (12) & $14(3)$ & $46(10)$ & $17(4)$ & $34(7)$ & $15(3)$ & $24(5)$ & $13(3)$ \\
\hline Not treated $\neq$ & $46(10)$ & $32(7)$ & 86 (19) & 69 (15) & 169 (37) & 124 & $213(46)$ & 178 (39) & 249 & 211 (46) & $283(61)$ & $283(53)$ & $315(68)$ & 273 (59) \\
\hline
\end{tabular}

* Day 1 defined as day before surgery. Day 2 is day of surgery before induction of anaesthesia. Days 3-8 are postoperative days.

†Because of heart rate between $55-65$ beats per minute and systolic blood pressure $\geq 100 \mathrm{~mm} \mathrm{Hg}{ }^{26}$

$\ddagger$ Đecause of contraindications (heart rate $<55$ beats per minute or systolic blood pressure $<100 \mathrm{~mm} \mathrm{Hg}$ ), discharge, or death. ${ }^{26}$

Table 5 No of reported serious adverse events

\begin{tabular}{|c|c|c|}
\hline & Metoprolol & Placebo \\
\hline \multicolumn{3}{|l|}{ Cardiovascular: } \\
\hline Myocardial infarction & 3 & 4 \\
\hline Myocardial ischaemia & 0 & 1 \\
\hline Congestive heart failure & 2 & $1^{*}$ \\
\hline Atrial fibrillation & 6 & 2 \\
\hline Atrioventricular dissociation & 2 & $1^{*}$ \\
\hline Bradycardia & 4 & 0 \\
\hline Hypotension & 2 & 1 \\
\hline Cardiac arrest & 2 & 1 \\
\hline Sudden death & 2 & 3 \\
\hline \multicolumn{3}{|l|}{ Pulmonary: } \\
\hline Respiratory failure & 1 & 0 \\
\hline Pneumonia & 1 & 1 \\
\hline \multicolumn{3}{|l|}{ Gastrointestinal: } \\
\hline Ileus & 1 & 1 \\
\hline Anastomosis leakage & 1 & 0 \\
\hline Gastrointestinal haemorrhage & 2 & 1 \\
\hline Perforated intestine & 0 & 1 \\
\hline Peritonitis & 1 & 0 \\
\hline Necrotising fasciitis & 0 & 1 \\
\hline Reoperation & 0 & 1 \\
\hline \multicolumn{3}{|l|}{ Cerebral: } \\
\hline Stroke & 2 & 0 \\
\hline Psychosis & 1 & 0 \\
\hline Delirium & 0 & 2 \\
\hline Dizziness & 0 & 1 \\
\hline Intoxication & 0 & 1 \\
\hline \multicolumn{3}{|l|}{ Metabolic: } \\
\hline Ketoacidosis & 1 & 0 \\
\hline \multicolumn{3}{|l|}{ Nephrological: } \\
\hline Acute tubular interstitial nephropathy & 1 & 1 \\
\hline \multicolumn{3}{|l|}{ Extremities: } \\
\hline Amputation & 0 & 1 \\
\hline \multicolumn{3}{|l|}{ Systemic: } \\
\hline Allergic reaction & 1 & 0 \\
\hline All & $36 \dagger$ & $26 \dagger$ \\
\hline
\end{tabular}

*One patient having two reported serious adverse events.

†No significant difference between metoprolol and placebo group, $\mathrm{P}=0.2$. heart rate by $11 \%(9 \%$ to $13 \%, \mathrm{P}<0.001)$ and mean arterial pressure by $3 \%(1 \%$ to $5 \%, \mathrm{P}<0.02)$. In two patients in the metoprolol group and five in the placebo group we discontinued the trial drug because treatment with $\beta$ blockers was indicated.

\section{Discussion}

The results of this trial showed that compared with placebo, metoprolol has no significant effect on short or long term outcomes in patients with diabetes undergoing major noncardiac surgery. This finding was consistent across the intention to treat and per protocol analyses as well as in analyses of the 496 patients with additional risk factors for coronary artery disease.

\section{Strengths and limitations}

This trial was a multicentre trial of the long term effects of metoprolol. We used adequate methods for generation of the allocation sequence and allocation concealment. ${ }^{14}$ We made efforts to maintain blinding through selection, treatment, monitoring, data management, and data analyses. Blinding is difficult in $\beta$ blocker trials because of changes in pulse and blood pressure, but the event committee performed blinded outcome assessment. ${ }^{10} \mathrm{We}$ used metoprolol in a sustained release formulation, comparable with atenolol, ${ }^{15}$ as long acting $\beta$ blockers offer better protection than short acting ones. ${ }^{16}$ Our trial is comparable with previous trials ${ }^{8}$ regarding intervention, dose, duration, the inclusion of patients at intermediate and high risk, surgery, and outcome measures, but we excluded patients already taking $\beta$ blockers. ${ }^{17} \mathrm{~A}$ total of 496 of our patients met the inclusion criteria used by Mangano et al, ${ }^{7}$ nearly two and a half times the sample size in that trial.

The potential limitations of this trial are that we included only patients with diabetes and we may not have included enough patients. However, $21 \%$ in the placebo group developed a primary outcome, enabling us to detect a hypothetical $7 \%$ absolute risk reduction with a power of $80 \%$, making our trial far more sensitive than previous trials. ${ }^{8}$ A daily dose of $100 \mathrm{mg}$ metoprolol controlled/extended release may not have ensured sufficient $\beta$ blockade, but heart rates and reductions in blood

Table 6 Means of heart rates and arterial pressures

\begin{tabular}{|c|c|c|c|c|}
\hline \multirow{2}{*}{ Day* } & \multicolumn{2}{|c|}{ Mean (SD, range) heart rate (beats/min) } & \multicolumn{2}{|c|}{ Mean (SD, range) arterial pressure ( $\mathrm{mm} \mathrm{Hg}$ ) } \\
\hline & Metoprolol & Placebo & Metoprolol & Placebo \\
\hline 1 & $78(12,47-120)$ & $79(12,50-128) \dagger$ & $103(14,58-150)$ & $104(14,61-145) \dagger$ \\
\hline 2 & $72(12,40-122)$ & $77(13,50-128) \ddagger$ & $99(14,58-147)$ & $102(14,63-145) \S$ \\
\hline 3 & $74(12,40-120)$ & $83(14,48-132) \ddagger$ & $91(16,56-139)$ & $94(16,50-149) \S$ \\
\hline 4 & $75(12,49-120)$ & $84(14,52-120) \ddagger$ & $92(15,51-149)$ & $95(15,47-132) \S$ \\
\hline 5 & $75(13,44-124)$ & $84(14,50-136) \ddagger$ & $92(14,58-140)$ & $96(14,58-142) \S$ \\
\hline 6 & $75(14,44-135)$ & $82(13,46-124) \ddagger$ & $93(14,55-144)$ & $96(14,63-148) \S$ \\
\hline 7 & $75(12,50-112)$ & $83(13,47-124) \ddagger$ & $94(14,53-127)$ & $97(15,50-167) \S$ \\
\hline 8 & $76(14,44-131)$ & $83(13,52-123) \ddagger$ & $92(14,51-149)$ & $97(15,57-140) \S$ \\
\hline
\end{tabular}

${ }^{*}$ Day 1 is first day of study drug administration on day before surgery. Day 2 is day of surgery. Days 3-8 are six postoperative days. Values days 1-8 are from just before next dose of study drug was to be given.

$+P=0.7$ at day 1 for comparison of mean heart rates and mean arterial pressures.

$\ddagger \mathrm{P}<0.001$ at day $2-8$ for comparison of mean heart rates.

$\S \mathrm{P}<0.02$ at day 2-8 for comparison of means of mean arterial pressures. 


\section{What is already known on this topic}

Guidelines recommend perioperative $\beta$ blockers for patients at cardiac risk who are undergoing major non-cardiac surgery, including those with diabetes

A meta-analysis of randomised trials concluded that perioperative $\beta$ blockade significantly reduces the perioperative burden of ischaemia but increases the number of episodes of bradycardia and hypotension compared with placebo

\section{What this study adds}

Compared with placebo, sustained release metoprolol (100 $\mathrm{mg}$ a day for up to eight perioperative days) given to patients with diabetes undergoing non-cardiac surgery does not affect long term mortality and cardiac morbidity

pressure were similar to those seen in previous trials. ${ }^{2}$ Initiating $\beta$ blockade with a higher dose in patients with cardiac risks before surgery may jeopardise the patients. In fact, several patients in this trial did not tolerate the full dose. Our intervention lasted longer than most of the trials included in the meta-analysis by Devereaux et al. ${ }^{8}$ We experienced fewer primary outcomes than expected during follow-up. Our analyses indicate that we cannot exclude a beneficial effect of $20 \%$ or less or a detrimental effect of $40 \%$ or less (table 2). Confounding by patients with a $\beta_{2}$ adrenergic receptor genotype ${ }^{18}$ is unlikely but possible.

\section{Comparison with related research}

Previous trials that showed a positive effect of perioperative $\beta$ blockers on morbidity and mortality may have overestimated the effect. $^{8}$ These trials had several methodological problems. ${ }^{814}$ Pooling the trials with low bias risk from the meta-analysis of Devereaux et al, ${ }^{8}$ the POBBLE trial, ${ }^{9}$ and the current trial showed no significant effect of $\beta$ blockers on 30 day perioperative myocardial infarction (relative risk $0.85,0.49$ to 1.46 ) or on 30 day mortality $(1.15,0.68$ to 1.95$)$. Even when we included a high bias risk trial ${ }^{2}$ in the analysis $\beta$ blockers did not significantly reduce 30 day mortality $(0.89,0.55$ to 1.43$)$. Admittedly, the $95 \%$ confidence interval leaves room for both benefit and harm. With 2112 patients randomised to perioperative $\beta$ blockade for non-cardiac surgery we may still observe both beneficial or harmful effects. ${ }^{19}$ In a retrospective study of 663635 patients, Lindenauer et al found that patients with the highest cardiac risk scores might benefit from perioperative $\beta$ blockade.$^{20}$ Patients with the lowest cardiac risk score and diabetes, however, might be harmed by $\beta$ blockade (odds ratios for death $1.28,1.10$ to 1.50$).{ }^{20}$ Our trial shows significant mortality and morbidity in patients with diabetes undergoing non-cardiac surgery, confirming previous observations. ${ }^{71121}$ Adjusment for preoperative insulin treatment in the multivariate analysis did not significantly affect the hazard ratio of metoprolol in our trial.

\section{Conclusions}

Our results show no significant effect of perioperative metoprolol on cardiac morbidity and mortality in patients with diabetes undergoing major non-cardiac surgery. The evidence is insufficient to recommend perioperative $\beta$ blockers for patients at risk of cardiac morbidity. It is premature for policy making organisations to use treatment with perioperative $\beta$ blocker as a measure of hospital quality. ${ }^{22}{ }^{23}$ Therapeutic actions ought to await the perioperative ischemic evaluation study (POISE) $)^{24}$ and systematic reviews.
We thank the patients who participated in the trial; our colleagues at the surgical departments for excellent collaboration; AstraZeneca for helpful discussions and excellent collaboration during the design and inclusion phase of the DIPOM trial and for supplying metoprolol and placebo; and John Wikstrand and Björn Karlson, AstraZeneca, Sweden, and Birgit Springer, AstraZeneca, Denmark, for helpful comments on an earlier draft. This trial was presented at the 2004 American Heart Association Scientific Sessions as a late breaking clinical trial and published as an abstract in $\mathrm{Cir}$ culation 2005;111:1725.Kim Winther Jacobsen, Anette Ulrich (Copenhagen University Hospital, KAS Herlev), Kristina Hartmann (Copenhagen University Hospital, KAS Glostrup), Henrik Seidelin, Anna Schnaberich (Copenhagen University Hospital, HS Bispebjerg Hospital, Christian Nielsen (Copenhagen University Hospital, Amager Hospital), Hikmet Karacan, Jan Lyderik (Copenhagen University Hospital, H:S Hvidovre Hospital, TB), Birgitte Tornkvist, Iben Foss Sorgenfrei, Anders Kyst (FAC Hillerød Hospital), Allan Horn, Lars Bo Svendsen (Copenhagen University Hospital, H:S Rigshospitalet, The Abdominal Centre), Anders Jensen, Ole Hendriksen (Copenhagen University Hospital, H:S Rigshospitalet, Gynecologic Centre) were clinical investigators. Cathrine Fabricius, Lone Andersen, Jette V Pedersen, and Pia Hughes were trial coordinators. Bitten Hansen, Mette Hansen, Ninna Frydendahl, Bessie Hødholdt and Karen Juliussen were secretaries; Cathrine Fabricius, Lone Andersen, Helle Bülow, Inger-Lise Poulsen, Annet Schack von Brockdorff, Kate Palle, Birgitte Rühmann, Søren Bang, Anette Bredsdorff, Lene Jensen, Kirstine Harbou, Jette Mieritz, Helle Kahl Andersen, Lene Thuesen, and Pia Andersen were study nurses. Per Hildebrandt (H:S Frederiksberg Hospital, Department of Cardiology), Sten Madsbad (H:S Hvidovre Hospital, Department of Endocrinology), and Tom Pedersen (Department of Anaesthesiology, H:S Bispebjerg Hospital) were on the event committee. Kristian Thygesen (Department of Cardiology, Århus County Hospital, Århus University Hospital), Jørgen Hilden and Ib Christiansen (Department of Biostatistics, Faculty of Health Sciences, Copenhagen University) were on the independent data monitoring and safety committee. Poul Staun-Olsen and Lisbeth Andreasen (Holbæk Hospital, Denmark) and Knut Borch-Johnsen and Britta Dragsfeldt (Steno Diabetes Center) did the serological tests.

Contributors: The DIPOM Trial Group consists of investigators from public hospitals in the greater Copenhagen area. Principal investigators were PN, KF, JW, RV, JJ, YM, PS, TC, LJ, JJ, GJ, NBF, KB, JBM, TB, and SY. Clinical investigators were MB, ABJ, AM, JR, M-BM, M-LR, PLP, RA, FP, MH, LLN JE, BD, DZ, AM, BØ, LPW, BK, GP, JW, and PW. NS developed the data management systems and was responsible for data management. PJ and JH carried out statistical analyses. ABJ (coordinating investigator and guarantor), CG, JW, AKE, GJ, and TC formed the steering committee.

Funding: AstraZeneca, Danish Heart Foundation, Danish Diabetes Foundation, Copenhagen Hospital Corporation's Research Council, Danish Medical Research Council's "Program for Strengthening Regional Collaboration within Medical Health Research," and Copenhagen Hospital Corporation. Competing interests: None declared.

Ethical approval: The trial was approved by the local ethics committee (journal No AGF/USS KA 99077ms), the Danish Medicines Agency (journal No AD-MET-0003), and the Danish Data Protection Agency.

1 Mangano DT, Browner WS, Hollenberg M, Li J, Tateo IM. Long-term cardiac prognosis following noncardiac surgery. The Study of Perioperative Ischemia Research Group. sis following noncardiac

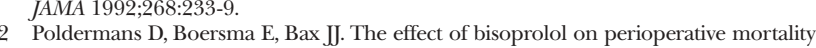
and myocardial infarction in high-risk patients undergoing vascular surgery. Dutch Echocardiographic Cardiac Risk Evaluation Applying Stress Echocardiography Study Group. N Engl J Med 1999;341:1789-94.

3 Ali MJ, Davison P, Pickett W, Ali NS. ACC/AHA guidelines as predictors of postoperative cardiac outcomes. Can J Anaesth 2000;47:10-9.

4 Goldman L, Caldera DL, Nussbaum SR. Multifactorial index of cardiac risk in noncardiac surgical procedures. N Engl J Med 1977;297:845-50.

5 Wallace A, Layug B, Tateo I. Prophylactic atenolol reduces postoperative myocardial ischemia. McSPI Research Group. Anesthesiology 1998;88):7-17.

6 Eagle KA, Berger PB, Calkins H, Chaitman BR, Ewy GA, Fleischmann KE, et al. ACC/ AHA guideline update for perioperative cardiovascular evaluation for non- cardiac surgery-executive summary. A report of the American College of Cardiology/ American Heart Association task force on practice guidelines (committee to update the 1996 guidelines on perioperative cardiovascular evaluation for noncardiac surgery). Circulation 2002;105:1257-67.

7 Mangano DT, Layug EL, Wallace A, Tateo I. Effect of atenolol on mortality and cardiovascular morbidity after noncardiac surgery. Multicenter Study of Perioperative Ischemia Research Group. N Engl J Med 1996;335:1713-20.

8 Devereaux PJ, Scott Beattie W, Choi PT-L, Badner NH, Guyatt G, Villar JC, et al. How strong is the evidence for the use of perioperative $B$ blockers in non-cardiac surgery? strong is the evidence for the use of perioperative B blockers in non-cardiac surgery?
Systematic review and meta-analysis of randomised controlled trials. BMJ 2005;331:313-21.

9 POBBLE Trial investigators. Perioperative beta-blockade (POBBLE) for patients undergoing infrarenal vascular surgery: results of a randomized double-blind controlled trial.J Vasc Surg 2005;41:602-9. 
10 Juul AB, Wetterslev J, Kofoed-Fnevoldsen A, Jensen G, Callesen T, Gluud C, The DIPOM Group. The diabetic postoperative mortality and morbidity (DIPOM) trial ationale and design of a multicenter, randomised, placebo-controlled, clinical trial of metoprolol for patients with diabetes mellitus who are undergoing major noncardiac surgery. Am Heart J 2004;147:677-83.

11 Juul AB, Wetterslev J, Kofoed-Enevoldsen A. Long-term postoperative mortality in diabetic patients undergoing major non-cardiac surgery. Eur J Anaesthesiol 2004;21:523-9.

12 Harris MI, Hadden WC, Knowler WC, Bennett PH. International criteria for the diagnosis of diabetes and impaired glucose tolerance. Diabetes Care 1985;8:562-7.

13 International conference on harmonisation of technical requirements for registration of pharmaceuticals for human use. ICH harmonised tripartite guideline for good clinical practice. 3rd ed. Richmond, Surrey: Brookwood Medical Publications, 2002.

14 Kjaergard LL, Villumsen J, Gluud C. Reported methodologic quality and discrepancies between large and small randomised trials in meta-analyses. Ann Intern Med 2001;135:982-9.

15 Omvik P, Leer J, Istad H, Westergren G. Equal efficacy and improved tolerability with 50 $\mathrm{mg}$ controlled-release metoprolol compared with $100 \mathrm{mg}$ conventional metoprolol in hypertensive patients. Am J Ther 1994;1:65-73.

16 Redelmeier DA, Scales DC, Kopp A. $\beta$ blockers for elective surgery in elderly patients: population based, retrospective cohort study. BMJ 2005:331:932

17 Shammash JB, Trost JC, Gold JM, Berlin JA, Golden MA, Kimmel SE. Perioperative beta-blocker withdrawal and mortality in vascular surgical patients. Am Heart $J$ 2001:141:148-53.

18 Lanfear DE, Jones PG, Marsh S, Gresci S, McLeod HL, Spertus JA. Beta 2 -adrenergic receptor genotype and survival among patients receiving beta-blocker therapy after an $5: 294: 1526-39$

19 Ioannidis J, Lau J. Evolution of treatment effects over time: emperical insight from recursive cumulative meta-analyses. Proc Natl Acad Sci USA 2001;98:831-6.

20 Lindenauer PK, Pekow P, Wang K, Mamidi DK, Gutierrez B, Benjamin EM. Perioperaive beta-blocker therapy and mortality after major noncardiac surgery. $N$ Engl Med 2005;353:349-61.

21 Huxley R, Barzi F, Woodward M. Excess risk of fatal coronary heart disease associated with diabetes in men and women: meta-analysis of 37 prospective cohort studies. BM 2006;332:73-8.

22 Siddiqui AK, Ahmed S, Delbeau H, Conner D, Mattana J. Lack of physician concordance with guidelines on the perioperative use of beta-blockers. Arch Intern Med 2004;164:664-7.

23 Lindenauer PK, Fitzgerald J, Hoople N, Benjamin EM. The potential preventability of postoperative myocardial infarction: underuse of perioperative beta-adrenergic blockde. Arch Intern Med 2004;164:762-6

24 Leslie K, Devereaux PJ. A large trial is vital to prove perioperative beta-blockade effectiveness and safety before widespread use. Anesthesiology 2004;101:803-6. (Accepted 13 March 2006)

bmj.com 2006;332:1482

Copenhagen Trial Unit, Centre for Clinical Intervention Research, H:S Rigshospitalet, Copenhagen University Hospital, Denmark

Anne Benedicte Juul research fellow

Jørn Wetterslev chief physician

Christian Gluud head of department

Per Winkel consultant statistician

Nader Salas data manager

Department of Internal Medicine, Kolding Fredericia Hospital, Denmark Allan Kofoed-Enevoldsen chief physician in endocrinology

Department of Cardiology and Anaesthesiology, H:S Hvidovre Hospital Copenhagen University Hospital, Denmark

Gorm Jensen head of department

Nikolai Bang Foss senior registrar in anaesthesiology

Jeanie Elkjær senior registrar in anaesthesiology

Bjørn Dehlie senior registrar in anaesthesiology
Department of Anaesthesiology, H:S Rigshospitalet, Copenhagen University Hospital, Denmark

Torben Callesen consultant in anaesthesiology

Lise Jørgensen senior registrar in anaesthesiology

Jørn Bo Madsen chief physician in anaesthesiology

Bjarne Øberg consultant in anaesthesiology

Boris Khanykin senior registrar in anaesthesiology

Stig Yndgaard consultant in anaesthesiology

Grazyna Perko senior registrar in anaesthesiology

Lars Peter Wang consultant in anaesthesiology

Department of Anaesthesiology, KAS Gentofte, Copenhagen University Hospital, Denmark

Peter Nørgaard consultant in anaesthesiology

Kim Fruergaard consultant in anaesthesiology

Morten Bestle consultant in anaesthesiology

Department of Anaesthesiology, KAS Herlev Hospital, Copenhagen University Hospital, Denmark

Rune Vedelsdal consultant in anaesthesiology

Andr Miran registrar in anaesthesiology

Department of Anaesthesiology, KAS Glostrup, Copenhagen University Hospital, Denmark

Jon Jacobsen consultant in anaesthesiology

Jakob Roed consultant in anaesthesiology

Maj-Britt Mortensen senior registrar in anaesthesiology

Pia Stjernholm consultant in anaesthesiology

Yvonne Mølgaard senior registrar in anaesthesiology

Department of Anaesthesiology, H:S Bispebjerg Hospital, Copenhagen University Hospital, Denmark

Jørgen Jørgensen senior registrar in anaesthesiology

Marie-Louise Rovsing senior registrar in anaesthesiology

Pernille Lykke Petersen senior registrar in anaesthesiology

Frank Pott senior registrar in anaesthesiolog

Merete Haas senior registrar in anaesthesiology

Rikke Albret senior registrar in anaesthesiology

Lise Lotte Nielsen senior registrar in anaesthesiology

Department of Anaesthesiology, Amager Hospital, Copenhagen University

Hospital, Denmark

Gun Johansson consultant in anaesthesiology

Department of Anaesthesiology, H:S Fredriksberg Hospital, Copenhagen

University Hospital, Denmark

Klavs Boysen consultant in anaesthesiology

Dusanka Zaric consultant in anaesthesiology

Anne Munksgaard senior registrar in anaesthesiology

Department of Anaesthesiology, FAC Hillerød, Hillerød, Denmark

Tine Blemmer senior registrar in anaesthesiology

Department of Biostatistics, Faculty of Health Sciences, Copenhagen University, Denmark

Jørgen Hilden statistician

Per Jensen statistician

Correspondence to: A B Juul anne.j@ctu.rh.dk 